\title{
Mechanical Characterization of the Human Atria Based on Patient- Specific Finite Element Method Models

\author{
Boris Nii $\mathrm{AS}^{1^{*}}$, Parente $\mathrm{ML}^{2}$ and Joao Manuel $\mathrm{RS}^{2}$
} \\ ${ }^{1}$ Department of Biomedical Engineering, University of Porto, Portugal \\ ${ }^{2}$ Department of Mechanical Engineering, FEUP, Rectory of the U.Porto Gomes Teixeira Square, Porto, Portugal
}

*Corresponding author: Sowah Boris Nii-Ako, MSc in Biomedical Engineering, University of Porto, Portugal, Tel: +351933538079; E-mail: borissowah@yahoo.co.uk

Received date: July 15, 2017; Accepted date: July 25, 2017; Published date: July 30, 2017

Copyright: () 2017 Boris Nii AS, et al. This is an open-access article distributed under the terms of the Creative Commons Attribution License, which permits unrestricted use, distribution and reproduction in any medium, provided the original author and source are credited.

\begin{abstract}
The left atrium (LA) is very essential in the overall performance of the cardiovascular process. Common cardiovascular diseases (CVD) of the atria are atrial fibrillation (AF) which is the most common cardiac arrhythmia in the world that affects over 9 million people in Europe per year and atrial stunning (AS) and can be resolved by minimal invasive cardiac interventions that accesses the LA. Trasseptal puncture technique (TSP) is the most used approach and the success rate depends on the experience of the surgeon. There are associated complications such as the possibility of multiple puncturing and procedural failures. To resolve these complications, the exact spot at which the septum requires to be traversed needs to be simulated and this project is aimed at developing technologies to assist physicians when performing this procedure. Finite element method (FEM) software called Abacus was employed to develop FEM model after effective analysis of the mechanical behavior of the atria specifically the atrial septal wall to enhance the effective optimization of the TSP technique.
\end{abstract}

Keywords: Atrial fibrillation; Cardiovascular diseases; Finite element method

\section{Introduction}

The human heart is a muscular organ which has four chambers, hollow and cone-shaped situated towards the left of the midline of the thoracic cavity. The atria are the upper two chambers divided by a wall-like structure called interatrial septum whiles the ventricles consist of the two lower chambers also divided by the interventricular septum. Globally the most common cause of death is CVD. In Europe, it is responsible for about $45 \%$ of all death which amounts to over four [1-4] million death per year with coronary heart disease (CHD) being the most common single cause of death forming about $19 \%$ and $20 \%$ of deaths in men and women respectively far more than deaths associated with cancer [1]. Since the four [4] chambers of the heart are independent, they thus have different associated cardaic diseases.

Evaluation of most heart diseases are done by the measurement of the LA pressure hence the need to be able to get access to the LA. Open chest surgery was initially used but it required a longer operational period and a long surgical cut in the chest organ hence the rapid rise of minimally invasive procedures which requires only a small puncture through the skin to access the vascular system and subsequently the LA. Minimally invasive technique has less post-operative pain, faster, involves less blood loss, less expensive and has a fewer procedural complications [2].

The objective of this dissertation is to study the quantification of the mechanical properties of the different tissues of the human atria and also to study the puncturing of the human atria using a needle during a TSP procedure to help improve the safety, efficiency and accuracy of the procedure.

\section{Fundaments and Related Works}

\section{Finite element method review}

FEM is a numerical approach by which many physical, engineering and scientific phenomena described in terms of partial differential equations are solved approximately [3]. It is used in solving engineering problems with complicated geometries, material properties and loadings. It is perceived to divide the physical phenomena into finite elements connected together by nodes. The higher the number of elements and nodes, the more accurate the solution. The strong form consists of the governing equations and the boundary conditions for the physical system while the weak form is obtained by restating the partial differential equations in an integral form. Discretization is the process of obtaining the finite number of discrete equations from the weak form.

Coupled Eulerian-Lagrangian (CEL) strives to combine the advantages of both the Lagrangian and Eulerian methods where the Eulerian material is tracked as it flows through the mesh by calculating the Eulerian volume fraction (EVF). CEL method involves mesh treatment and surface reconstruction as well as solving the coupled fluid and structure equations.

\section{Biomechanical simulation}

Novel biomechanical models relevant to clinical practice have been achieved due to the recent advances in non-invasive high-resolution image acquisition techniques making them feasible to generate accurate patient-specific anatomical models. Some notable instances are the proposed framework to simulate the procedure used to treat mitral valve (MV) regurgitation using a minimally invasive cardiac procedure and the developed a patient-specific models to quantify and characterize the interaction between the trans-catheter stent and the stenotic aortic valve (SAV) [4]. 


\section{Simulation of the atrium}

Moyer et al. constructed and validated a FEM model of the normal human left atrium using anatomic information obtained from cardiac MRI, iterative algorithm to estimate unloaded and material properties as well as fiber orientations derived from published articles [5]. The development of a realistic 3D computer model of the human atria to examine the relationship between different atrial arrhythmic propagation patterns and the EGM observed at different points along the atrial surface.

\section{Simulation Methodology}

\section{Needle geometry and modeling}

The lower the insertion force, the lower the needle deflection and tissue deformation during the needle insertion process [6] and also a higher accuracy. The optimized needle cutting-edge model developed by Yancheng et al. [7] was developed using solid works software. The developed needle had a bevel length of $13.30 \mathrm{~mm}$ and a bevel angle of $12.4^{\circ}$.

\section{Tissue geometry and modeling}

To model the soft tissue and the cardiac muscle of the heart, two different Eulerian patches with two layers were developed using the Abacus software with dimensions of $40 \mathrm{~mm}$ by $20 \mathrm{~mm}$ and a modified patch $40 \mathrm{~mm}$ by $40 \mathrm{~mm}$ to evaluate the effect of varying the size of the patch. The model comprised of the tender tissue and the soft tissue for the soft tissue patch and the tender tissue and cardiac muscle for the cardiac muscle patch. The tender tissue had a Young's modulus of 0.129 $\mathrm{MPa}$ and that of the soft tissue was $0.007 \mathrm{MPa}$ while that of the cardiac muscle is $0.1 \mathrm{MPa}$. A Poisson ratio of 0.475 was used. The tender tissue was designed to occupy the top- most region of the assembly and had a depth of $5 \mathrm{~mm}$ while the soft tissue or cardiac muscle occupied the remaining part of the Eulerian model.

\section{Simulation of the needle insertion}

Two different sets of simulations were performed; needle insertion of the soft tissue and that of the cardiac muscle. The mesh of the model was generated by parts and not collectively due to the complex geometry of the needle. The needle was rotated at different angles $\left(15^{\circ}\right.$, $30^{\circ}, 45^{\circ}, 60^{\circ}$ and $90^{\circ}$ ) with respect to $\mathrm{X}$ and $\mathrm{Z}$ axis and their respective displacement co- ordinates were calculated to perform the simulation.

\section{Results and Discussion}

Three sets of results were obtained from the simulation; a) the needle insertion forces $b$ ) varying the size of the patch and c) Mises stress distribution. With the needle insertion forces, five sets of simulations were performed for the rotation of the needle about the $\mathrm{X}$ and $\mathrm{Z}$-axes for both the soft tissue and cardiac muscle patch. With the rotation about the $\mathrm{Z}$-axis for the soft tissue patch, the highest insertion force was at the $60^{\circ}$ with $0.5 \mathrm{~N}$ and the lowest at the $30^{\circ}$ with $0.377 \mathrm{~N}$ while the highest insertion force was at $15^{\circ}$ with $0.88 \mathrm{~N}$ and the lowest was at $60^{\circ}$ with $0.272 \mathrm{~N}$ for the rotation about the X-axis. With the cardiac muscle patch, the highest force was at $45^{\circ}$ with $1.94 \mathrm{~N}$ whereas the lowest force was at $90^{\circ}$ at $1.52 \mathrm{~N}$ for the rotation about the Z-axis while the highest force was at $15^{\circ}$ with $2.45 \mathrm{~N}$ whereas the lowest force was at $60^{\circ}$ rotation with $0.59 \mathrm{~N}$ for the rotation about the $\mathrm{X}$-axis. With varying the size of the patch, the graphs of the simulation of the new dimensions of the soft tissue patch about the $30^{\circ}$ rotation about the $\mathrm{X}$ and Z-axes was similar to that of the initial patch. The distinct difference is that the bigger soft tissue patch had lower maximum needle insertion force compared to that obtained from the initial size. With the $45^{\circ}$ about the Z-axis, the graphs differed slightly from the initial size of the soft tissue patch. It also had a higher force of $0.5 \mathrm{~N}$ compared to that of the initial size which was $0.41 \mathrm{~N}$. The graphs of the new dimension of the cardiac muscle about the $30^{\circ}$ rotation about the $\mathrm{Z}$ and $\mathrm{X}$-axes were very similar to the initial patch. The obvious difference was that the bigger dimension had lower maximum force compared to that of the initial patch and is it very prominent in the Xaxis rotation. The $45^{\circ}$ rotation about the $\mathrm{Z}$-axis had similar graph and maximum forces as the initial cardiac muscle patch. With the $45^{\circ}$ rotation about the $\mathrm{X}$-axis, the graph differed largely from that obtained from the initial patch. It also had a higher force of $1.49 \mathrm{~N}$ compared to the initial patch size which was $1.06 \mathrm{~N}$. As expected, the Mises stress distribution revealed that the maximum Mises stress coincided with the peak of the graphs of the needle insertion force against time indicating that the highest forces were experienced at the highest peaks of the graph for both materials of the model.

\section{Conclusion}

The cardiac muscle simulation had higher insertion forces compared to the soft tissue patch as expected due to the higher mechanical properties of the cardiac muscles. The size of the tissue and cardiac muscle patch may/or may not have significant effects on the results based on the angle of rotation as well as the axis of rotation as seen in the earlier results. All the graphs had at least two spikes since two layers of different materials of different mechanical properties were used.

In as much as this research work had many positives, there was no mesh convergence study conducted for the generated finite element mesh for the needle.

This research work can be modified by developing the actual anatomical structure of the human atria and simulating the needle insertion procedure using the CEL method to obtain more realistic results and simulations.

\section{References}

1. Townsend N, Nichols M, Scarborough P, Rayner M (2015) Cardiovascular disease in Europe - Epidemiological update 2015. Eur Heart J 36: 2696-2705

2. Rosengart TK, Feldman T, Borger MA, Vassiliades TA, Gillinov AM, et al. (2008) Percutaneous and minimally invasive valve procedures - A scientific statement from the American Heart Association Council on Cardiovascular Surgery and Anesthesia, Council on Clinical Cardiology, Functional Genomics and Translational Biology Interdisciplina. Circulation 117: 1750-1767.

3. Fish J (2007) A first course in finite elements. John Willey and Sons Ltd, USA.

4. Wang Q, Sirois E, Sun W (2012) Patient-specific modeling of biomechanical interaction in transcatheter aortic valve deployment. J Biomech 45: 1965-1971.

5. Moyer CB, Norton PT, Ferguson JD, Holmes JW (2015) Changes in global and regional mechanics due to atrial fibrillation: Insights from a coupled finite-element and circulation model. Ann Biomed Eng 43: 1600-1613.

6. Podder T, Sherman J, Rubens D, Messing E, Strang J, et al. (2008) Methods for prostate stabilization during transperineal LDR brachytherapy. Phys Med Biol 53: 1563- 1579. 
Citation: Boris Nii AS, Parente ML, Joao Manuel RS (2017) Mechanical Characterization of the Human Atria Based on Patient-Specific Finite Element Method Models. J Bioengineer \& Biomedical Sci 7: 233. doi:10.4172/2155-9538.1000233

Page 3 of 3

7. Wang Y, Chen RK, Tai BL, McLaughlin PW, Shih AJ (2014) Optimal needle design for minimal insertion force and bevel length. Med Eng Phys 36: 1093-1100. 